\title{
La mécanique des biofilms à la surface de liquides
}

\author{
Carine Douarche ${ }^{(1)}$ (carine.douarche@u-psud.fr), Virginie Bailleux ${ }^{(1)}$, Catherine Even $^{(1)}$, \\ Jean-Marc Allain ${ }^{(2,3)}$, Christophe Regeard(4) et Éric Raspaud( ${ }^{(1)}$ (eric.raspaud@u-psud.fr) \\ (1) Laboratoire de Physique des Solides (CNRS UMR8502, Université Paris-Sud, \\ Université Paris-Saclay), 91405 Orsay \\ (2) Laboratoire de Mécanique des Solides (École polytechnique et CNRS), \\ 91128 Palaiseau Cedex \\ (3) M3DISIM, INRIA, Université Paris-Saclay, Campus de l'École polytechnique, \\ 91120 Palaiseau \\ (4) Institut de Biologie Intégrative de la Cellule (I2BC), CEA-CNRS-Université Paris-Sud, \\ 91198 Gif sur-Yvette
}

Les micro-organismes peuvent

coloniser les surfaces

environnantes et s'organiser

en un film de plusieurs centaines

de microns d'épaisseur, appelé

« biofilm ». Ils sécrètent une

matrice extracellulaire polymérique

qui assure une véritable cohésion

et protection physique de la colonie,

avec des effets qui peuvent être

aussi bien bénéfiques que mortels

pour son environnement.

Nous nous sommes intéressés

aux forces mécaniques structurant

cette matière vivante solide

et contribuant à maintenir

son intégrité. Nous avons travaillé

sur des biofilms de bactéries

flottant à la surface d'un liquide.

Nous montrons que ces systèmes

ont des propriétés mécaniques

originales et remarquables,

du fait de leur capacité à croître

et proliférer.

\section{Introduction aux biofilms}

Depuis la découverte des biofilms, la vision des biologistes sur le monde microbien a radicalement changé. En effet, les microorganismes (bactéries, archées, champignons, protistes, virus...) et, plus particulièrement, les bactéries, ont été pendant longtemps uniquement considérés comme existant sous une forme dite "planctonique ", c'est-à-dire en suspension dans l'eau. Or, la vie microbienne de notre planète a colonisé non seulement les milieux aqueux mais également tous les milieux solides. Il est donc rare de trouver des surfaces totalement dépourvues de microbes, autre nom des micro-organismes. On estime d'ailleurs que $80 \%$ de la masse microbienne sur Terre existe sous une forme adhérée pouvant potentiellement donner naissance à un biofilm (fig. 1). Sachant que les microorganismes représentent plus de 90\% des espèces et $60 \%$ de la biomasse sur Terre, cela place les biofilms comme le mode de vie principal sur la planète. Dans la nature, les biofilms ont été observés dans des niches écologiques aussi variées que la surface des plantes, les sols, les interfaces air-eaux stagnantes, les sédiments marins, la peau et les microbiotes des animaux, etc. La plupart du temps, ces biofilms naturels comportent plusieurs espèces.

Tous les biofilms ont pour point commun une cohésion des cellules assurée grâce à une matrice extracellulaire. Celle-ci est composée de polymères complexes (souvent dérivés de polysaccharides), d'eau et quelquefois de protéines et d'acides nucléiques. Elle constitue un véritable rempart protecteur contre l'environnement souvent hostile et confere au biofilm des propriétés de matière solide. La grande majorité des biofilms sur Terre joue un rôle important dans le fonctionnement de la planète et intervient dans les cycles de la matière, le renouvellement des sols, l'assimilation des aliments chez les animaux, etc. Les exemples de biofilms "bénéfiques " sont pléthore. A contrario, lorsqu'une bactérie pathogène se développe en biofilm, elle devient plus résistante aux traitements antibiotiques et elle est capable de coloniser facilement des implants médicaux, ce qui représente la cause principale des infections nosocomiales. La compréhension de tous les processus biologiques, génétiques, chimiques ou physiques impliqués dans la formation des biofilms est donc capitale pour mieux les utiliser ou mieux les combattre. Les physiciens ont ainsi leur mot à dire.

Nous nous sommes intéressés aux propriétés mécaniques des biofilms. Nous avons choisi de travailler sur des biofilms flottant à la surface d'un liquide plutôt qu'adhérant sur une surface solide, afin de distinguer clairement la réponse mécanique du biofilm solide de celle du substrat liquide. Les biofilms étudiés sont formés par une souche sauvage (nommée NCIB 3610) de la bactérie du sol Bacillus subtilis, cette bactérie servant souvent de modèle de laboratoire. Une des premières questions que nous nous sommes posées concerne l'existence de contraintes internes (appelées encore " précontraintes "), c'est-à-dire de contraintes existant dans le matériau alors 


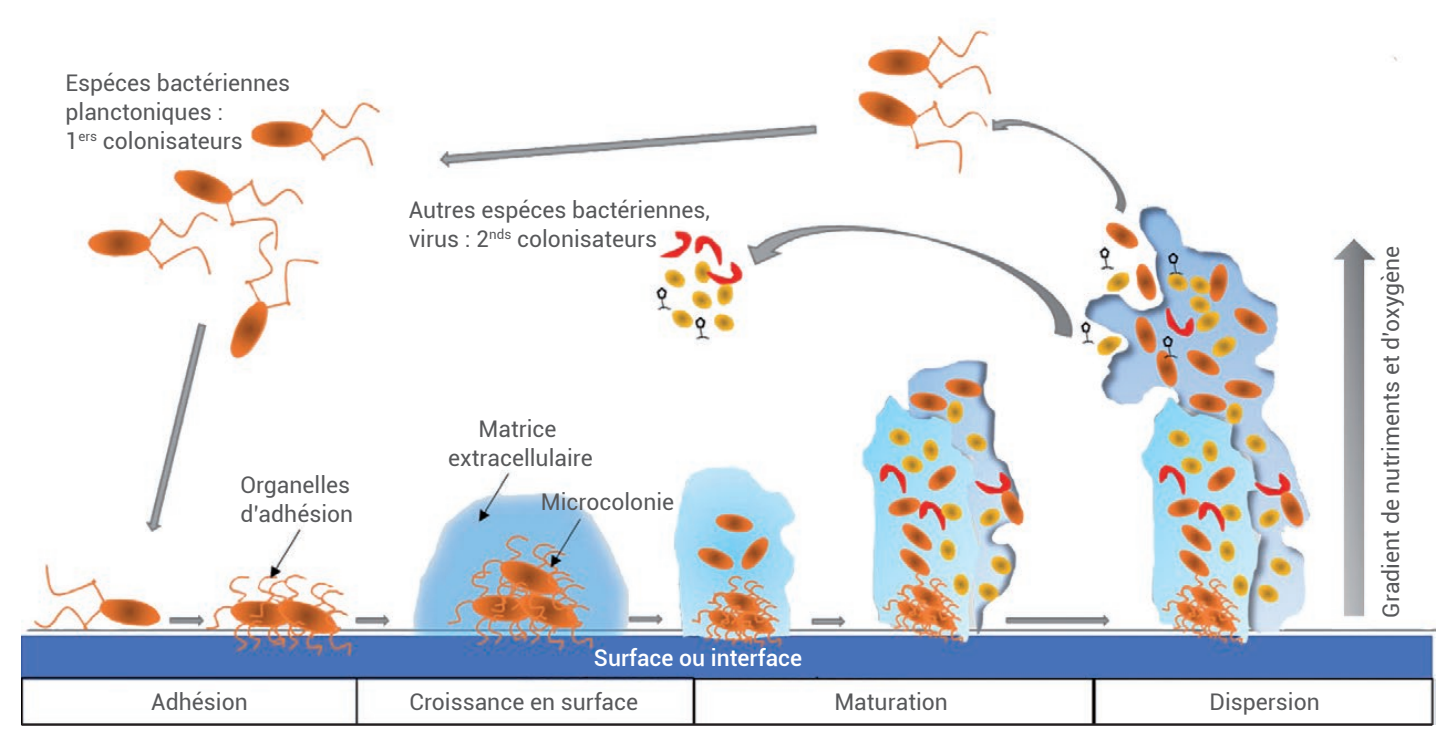

1. Les différentes étapes de formation d'un biofilm. Un biofilm peut être défini comme une communauté multicellulaire colonisant une surface ou une interface et dans laquelle les cellules (notamment les bactéries) sécrètent de la matrice extracellulaire polymérique. Sa formation est généralement décrite comme une succession d'étapes : adhésion, croissance en surface, maturation puis dispersion.

\section{Contraintes internes dans les matériaux}

Une contrainte (stress en anglais) est une force par unité de surface s'exerçant localement sur les parois d'un petit volume de matériau. Elle peut avoir n'importe quelle orientation par rapport à la surface et, d'une manière

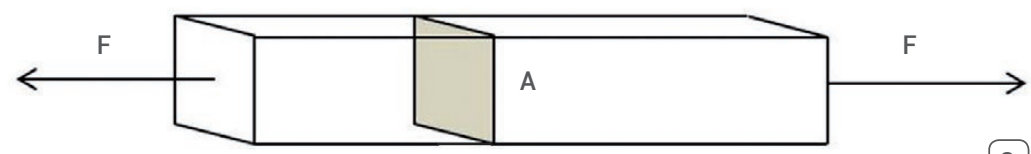
générale, on peut la décomposer en deux composantes : normale et tangentielle. La contrainte normale met le matériau en tension (les particules du matériau tirent les unes sur les autres) ou en compression (les particules poussent les unes sur les autres). La contrainte tangentielle cisaille quant à elle le matériau.

Prenons l'exemple (fig. E1a) d'un barreau mis en tension par deux forces égales et opposées

(de norme $F$ ) appliquées à ses deux extrémités : n'importe quelle section d'aire A perpendiculaire à l'axe du barreau subit alors une contrainte en tension $\sigma=F / A$. Cette contrainte traduit l'équilibre mécanique local entre deux sous-parties du barreau, situées de chaque côté de la section. Si le barreau est en trop forte compression, il finira par flamber, c'est-à-dire par se courber tout en restant élastique : un bon exemple est une règle que l'on courbe en appuyant assez fort des deux côtés (et qui revient quand on relâche la force).

On parle de " contrainte interne » ou de " précontrainte " (prestress en anglais) lorsque la contrainte considérée existe en l'absence de force extérieure. Le béton précontraint, illustré sur la figure E1b, est un exemple connu car il est largement utilisé dans la construction de bâtiments. La compression du béton est réalisée en mettant en traction une armature d'acier située à l'intérieur de la poutre. La précontrainte de compression le rend plus résistant aux forces qu'il subit. Dans le domaine de l'ingénierie des matériaux, la trempe du verre ainsi que le grenaillage de pièces métalliques permettent aussi d'améliorer leurs propriétés mécaniques : le traitement génère une précontrainte de compression qui améliore la tenue mécanique de leur surface en augmentant leur résistance aux initiations et propagations de fissures. De nombreux matériaux vivants ont également des contraintes internes. Celles-ci apparaissent clairement lorsque qu'on découpe un morceau du matériau : il change alors de forme sous l'effet des contraintes internes qui se libèrent. On peut citer un certain nombre de plantes (la rhubarbe ou la pastèque par exemple), de tissus, d'organes (comme les artères) : les contraintes internes proviennent de leur croissance dans un environnement complexe, par exemple lorsqu'un tissu grandit plus vite que le tissu voisin. 
qu'aucune force extérieure ne lui est appliquée (voir l'encadré p. 21). En effet, celles-ci apparaissent dans de nombreux systèmes biologiques en croissance et sont aussi présentes dans maints objets du quotidien. Comme elles permettent de rendre les matériaux plus résistants à une force mécanique extérieure, nous nous sommes demandé si de telles contraintes existaient dans les biofilms.

\section{Le biofilm : un matériau précontraint}

Notre dispositif expérimental est présenté sur la figure 2a [1]. La croissance du biofilm a lieu dans un récipient rectangulaire délimité par quatre parois verticales. Une paroi mobile reliée à un capteur de force est maintenue plongée au centre, dans le milieu de culture, pendant la croissance du biofilm. Le capteur mesure la composante normale à la surface de la paroi et une valeur nulle de la force sur le capteur correspond à une situation où toutes les forces qui agissent sur cette paroi sont nulles ou bien se compensent.

Après environ deux jours d'incubation, le biofilm forme une pellicule plane qui recouvre toute la surface du liquide et adhère à toutes les parois verticales. Dans cet état initial, le biofilm entoure la paroi mobile. Pour mettre en évidence la contrainte interne, nous avons réalisé une série de découpes à l'aide d'un scalpel, comme illustré par les lignes et les flèches colorées de la figure $2 \mathrm{~b}$. Les valeurs correspondantes de la force à chaque étape sont tracées en figure 2c. À l'étape 1 (en bleu), le biofilm est intact et aucune force n'est détectée. À l'étape 2 (lignes fléchées de découpe en noir), nous avons enlevé la partie du biofilm qui se trouve derrière la paroi mobile. Il apparaît alors une force négative sur le capteur ; cette force, illustrée par une flèche en figure $2 a$, pousse sur le capteur ce qui montre l'existence d'une force compressive dans le biofilm. À l'étape 3 (en vert), nous avons découpé le biofilm en suivant les deux lignes fléchées vertes, ce qui laisse une bande de biofilm de largeur égale à celle de la paroi mobile. La force résultante est alors un peu moins élevée en valeur absolue, mais toujours négative. Nous avons enregistré cette valeur de force compressive dans toutes nos expériences. Enfin, nous avons détaché la pellicule du bord opposé en suivant la ligne fléchée violette, et écarté la paroi mobile du bord opposé pour que la pellicule puisse avoir plus d'espace pour relaxer. La force redevient alors nulle (étape 4, en violet). Toutes les mesures indiquent ainsi qu'il existe une force de compression dans le biofilm et qu'elle est due au confinement du biofilm dans la boîte. À noter que cette force est bien présente dans le biofilm intact de l'étape 1 mais elle est contrebalancée par une force opposée qui s'exerce sur l'autre face de la paroi mobile; la résultante des deux, mesurée en figure 2c, devient alors nulle.

Nous avons mesuré la force compressive à l'étape 3 sur différents échantillons ayant des durées d'incubation différentes (de quelques heures à plusieurs jours). Nous observons que la force mesurée est nulle avant la formation d'une pellicule, augmente puis devient constante dès qu'un biofilm apparait. Cette valeur reste constante quel que soit l'âge du biofilm, ce qui montre que les bactéries construisent un biofilm en maintenant la précontrainte constante. Lorsque la force mesurée est divisée par la surface de la section de biofilm sollicitée (épaisseur du biofilm de l'ordre de 300 micromètres multipliée par la largeur de la paroi mobile), nous obtenons une valeur de la contrainte interne de l'ordre de - $80 \mathrm{~Pa}$. Cette faible valeur reste constante et est indépendante des dimensions macroscopiques du système (distance entre paroi mobile et bord opposé d'une part, et largeur de la paroi mobile d'autre part). Nous pouvons supposer que le même type de contrainte apparaitra dans un biofilm poussant sur une surface plane solide ou dans un biofilm présentant des hétérogénéités ou des taux de croissance différents à l'intérieur de celui-ci.

Le biofilm étant précontraint, nous avons voulu vérifier si cette précontrainte permettait au biofilm de mieux résister à des dommages éventuels. Lorsque nous essayons de détériorer avec une pointe de pipette une pellicule compressée, la contrainte interne tend à refermer la pellicule dans son état initial ; la cohésion et l'intégrité du biofilm sont ainsi maintenues (voir la vidéo [2]). Ce n'est pas le cas si la contrainte interne est nulle et que la pellicule est complètement relaxée (voir la vidéo [3]).
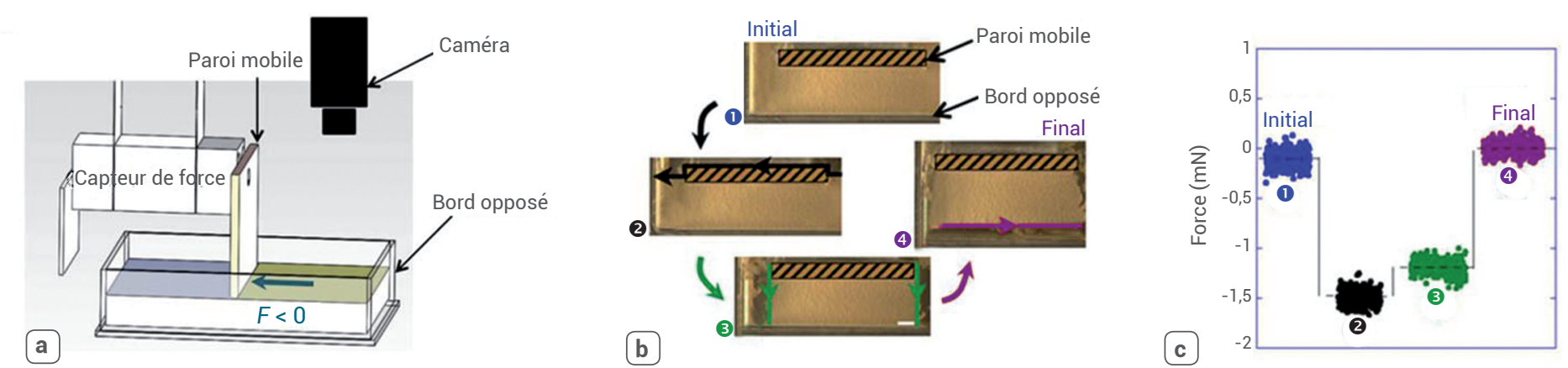

2. Mise en évidence de contraintes internes dans un biofilm.

(a) Vue schématique en perspective du dispositif. La vue illustre l'état de la pellicule à l'étape de découpe numérotée 2 en (b), une fois que la partie de biofilm située à gauche de la paroi mobile sur la figure a été retirée de l'échantillon. Une force négative F, schématisée par une flèche, correspond au cas où la plaque s'éloigne du bord opposé.

(b) Vues de dessus du biofilm au cours des étapes de découpe. La plaque mobile est schématisée par un rectangle hachuré et les lignes fléchées colorées montrent les découpes successives. Barres d'échelle $=5 \mathrm{~mm}$.

(c) La force agissant sur la plaque mobile est mesurée à chaque étape de découpe (bleu : étape 1, noir : 2, vert : 3, violet : 4). [1]. 


\section{La morphologie ridée des pellicules}

En d'autres termes, indépendamment de la géométrie de l'expérience, il existe un phénomène de régulation au cours de la croissance du biofilm, qui maintient constante la contrainte interne qu'il développe. Étonnamment, cette contrainte " critique " apparaît assez tôt dans la vie du biofilm. Ainsi, lorsqu'il va grandir et croître dans un espace limité, la contrainte va atteindre cette valeur critique et la pellicule n'aura pas d'autre choix que de se déformer en dehors du plan de l'interface eau-air et de former une structure ridée telle celles observées sur la figure 3 [4]. Comme le montre cette figure, la morphologie varie d'une souche à l'autre. Elle peut aussi varier en fonction du milieu de culture. La différence de morphologie provient de plusieurs facteurs comme la composition de la matrice extracellulaire ou l’hétérogénéité du biofilm.

En étudiant en détail les motifs si particuliers formés par les biofilms à maturation de la souche NCIB 3610 (vue de droite en figure 3), nous nous sommes rendus compte qu'il existe une longueur caractéristique des rides dans le système, de l'ordre d'un ou deux millimètres, et très reproductible d'un biofilm à l'autre. Ce phénomène, dit de "flambage ", bien connu dans le cas de matériaux élastiques sous compression, correspond à une instabilité mécanique et induit une ondulation du matériau. La longueur d'onde caractéristique prédite théoriquement résulte alors d'une compétition entre l'énergie de flexion du matériau et l'énergie associée à la déformation du substrat liquide (l'énergie gravitationnelle pour une surface ondulée); nous avons trouvé que la prédiction théorique de ce modèle est du même ordre de grandeur que la valeur millimétrique mesurée expérimentalement.

À noter que des rides de plus grande amplitude peuvent aussi apparaitre dans certains biofilms, comme ceux de la souche sauvage DV1 (figure 3 de gauche). Il s'agit dans ce cas de plis dont la formation permet de localiser la déformation du biofilm en quelques endroits précis, c'est-à-dire en leurs sommets, le reste du biofilm pouvant être quasiment plat.
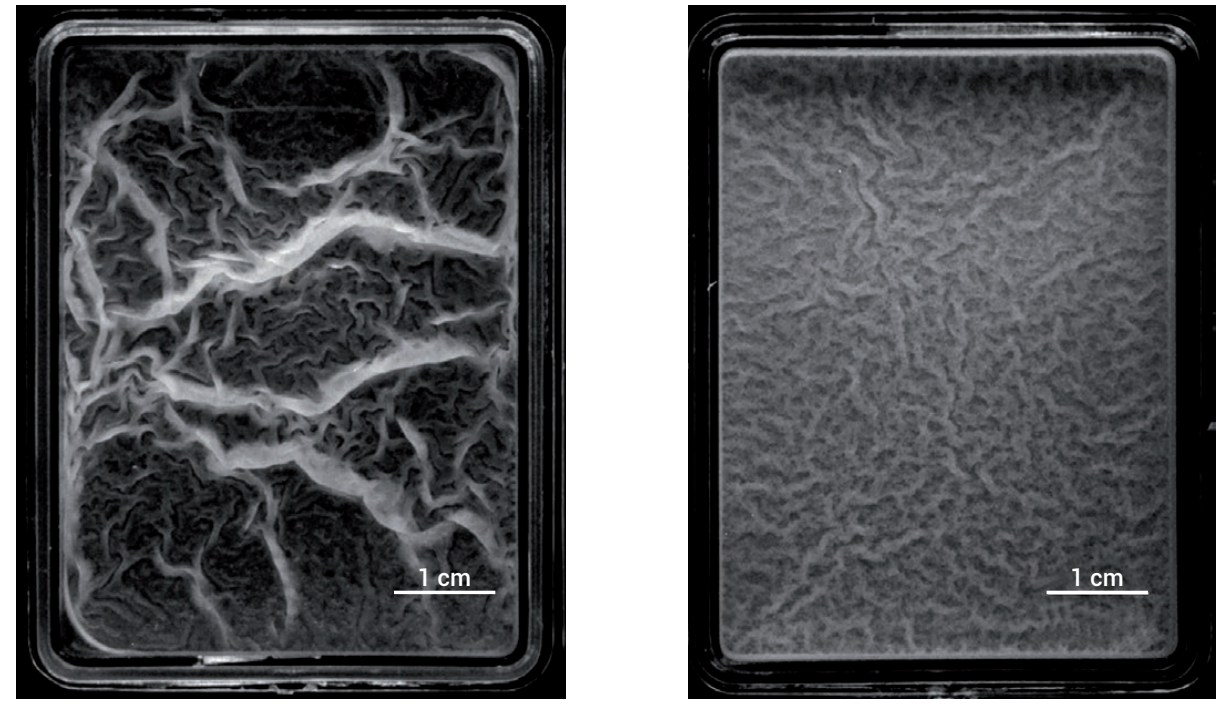

3. Formation de rides dans des biofilms en compression. Vue de dessus de deux biofilms de Bacillus subtilis de type sauvage flottant sur le milieu de culture liquide après environ 67 heures d'incubation à $23^{\circ} \mathrm{C}[4]$.

À gauche : la souche sauvage DV1. À droite : la souche sauvage NCIB 3610 .

\section{La rhéologie}

L'apparition de rides nous permet de supposer un comportement élastique des biofilms de B. subtilis. Pour le mettre en évidence, nous avons légèrement modifié le montage expérimental décrit précédemment, de façon à solliciter mécaniquement de manière contrôlée le biofilm. Cette fois-ci, deux parois mobiles sont maintenues plongées dans le milieu de culture au cours de la croissance du biofilm (fig. 4a). L'une est reliée à un capteur de force comme précédemment et l'autre à une platine pouvant se déplacer selon l'axe normal à la surface des parois. Ainsi, il est possible d'augmenter la distance entre les deux parois et d'imposer alors une déformation au biofilm (qui adhère aux deux parois). Dans l'expérience présentée en figure 4b, la déformation imposée au biofilm augmente linéairement au cours du temps, jusqu'à ce que le biofilm se rompe complètement après 60 secondes. Après rupture, la paroi reliée à la platine continue de se déplacer librement sans connexion avec le biofilm. Pendant le déplacement de la paroi, nous mesurons simultanément la force sur le capteur. Afin de simplifier l'interprétation de la mesure, nous gardons uniquement la bande de biofilm qui se trouve entre les deux parois, c'est-à-dire que nous nous arrêtons à l'étape 3 des schémas de découpe illustrés en figure 2a. En mesurant l'épaisseur initiale du biofilm et connaissant sa largeur, nous pouvons alors représenter une contrainte en fonction du temps (courbe du bas de la figure $4 \mathrm{~b}$ ) ou en fonction de la déformation imposée $[4,5]$. La contrainte augmente au cours du temps jusqu'à la rupture du biofilm, puis devient nulle au-delà puisque le biofilm détaché n'est plus confiné ni soumis à aucune force extérieure.

Avant rupture, la figure $4 \mathrm{~b}$ montre très clairement que la gamme de contraintes négatives (en compression) est au moins aussi étendue que la gamme de contraintes positives (en tension). Les deux régimes de compression et de tension sont tout aussi importants l'un que l'autre pour décrire les propriétés mécaniques du biofilm. Le domaine des petites déformations réversibles se trouve ainsi dans le régime de compression et, dans ce domaine, la variation de la contrainte est linéaire avec la déformation, impliquant un comportement élastique du matériau. Le module élastique (paramètre physique indiquant la capacité d'un matériau à se déformer sous la contrainte) est en moyenne de l'ordre de $300 \mathrm{~Pa}$, révélateur d'un matériau particulièrement "mou ". Cette valeur est en effet très faible par rapport aux modules élastiques de matériaux classiques comme le caoutchouc (de l'ordre de $10^{6} \mathrm{~Pa}$ ), mais reste comparable aux modules de cellules eucaryotes ou de tissus/organes mous comme le cerveau (de l'ordre de $10^{3} \mathrm{~Pa}$ ). Cette valeur dépend de la structure du biofilm ainsi que de la composition de la matrice extracellulaire secrétée par les bactéries. Le domaine des grandes déformations 
\〉

irréversibles menant à la rupture se trouve dans le régime de tension [5]. La déformation devient alors plastique et non uniforme, certaines parties se déformant plus que d'autres. Finalement, nous pouvons supposer que le biofilm tire profit de ces propriétés rhéologiques étonnantes, car un morceau de biofilm peut ainsi facilement se détacher sous l'effet d'une petite perturbation et coloniser un autre espace, la dispersion étant une étape importante dans la vie bactérienne (fig. 1).

\section{Conclusion}

Au cours de leur croissance, les biofilms développent des propriétés mécaniques étonnantes et cruciales à leur survie en milieu naturel. La présence d'une contrainte interne de compression, pourtant de faible intensité, permet de maintenir la cohésion du biofilm et la proximité entre les microorganismes. La contrainte augmente au cours de la formation du biofilm jusqu'à atteindre une valeur critique à partir de laquelle il flambe. Ainsi, une morphologie ridée apparaît, augmentant la surface d'échange du biofilm avec le milieu sur lequel il se développe.
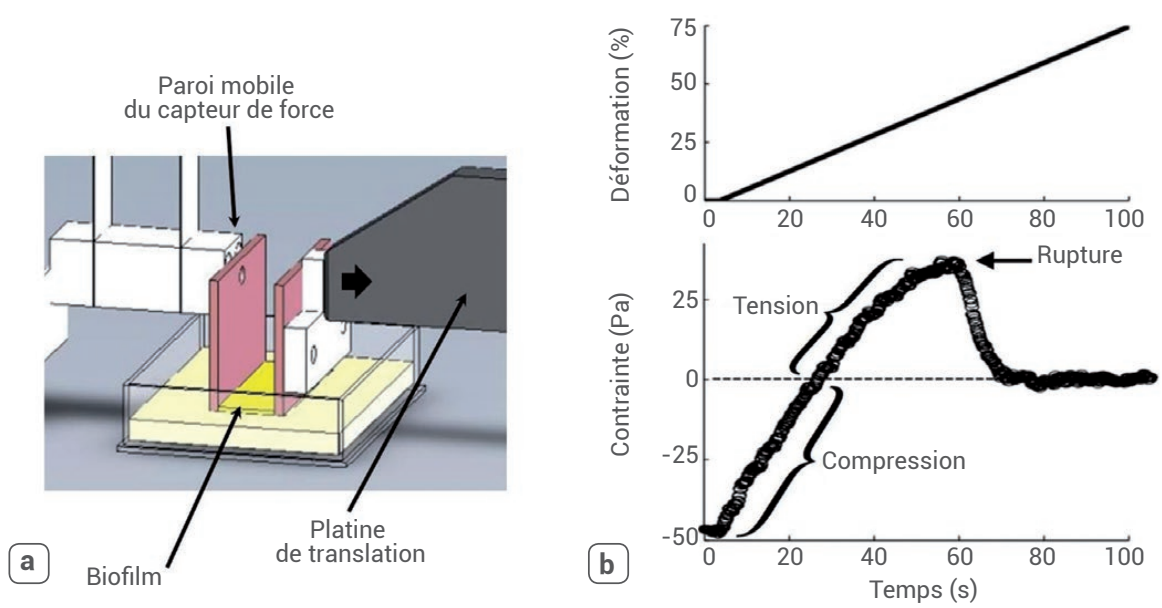

4. Déformation et rupture d'un biofilm sous traction.

(a) Schéma du montage expérimental permettant de sonder les propriétés mécaniques d'un biofilm flottant : lors de son développement, le biofilm s'accroche aux deux plaques plongées dans la solution. L'une est reliée à une platine de translation et l'autre à un capteur de force. (b) Nous imposons une déformation relative qui augmente linéairement au cours du temps (courbe du haut) et nous mesurons simultanément l'évolution de la contrainte induite par cette déformation (courbe du bas). [5].

\section{Références}

1• C. Douarche, J.-M. Allain, É. Raspaud, Biophys. J., 109 (2015) 2195-2202.

2• www.cell.com/cms/attachment/2092787303/ 2076627792/mmc3.mp4

3. www.cell.com/cms/attachment/2092787303/ 2076627791/mmc4.mp4
4. M. Trejo, C. Douarche, V. Bailleux, C. Poulard, S. Mariot, C. Regeard, É. Raspaud, Proc. Natl. Acad. Sci. U.S.A., 110 (2013) 2011-2016.

5• E.C. Hollenbeck, C. Douarche, J.-M. Allain, P. Roger, C. Regeard, L. Cegelski, G.G. Fuller, É. Raspaud, J. Phys. Chem. B, 120 (2016) 6080-6088. 